\title{
Developing new locality results for the Prüfer Code using a remarkable linear-time decoding algorithm
}

\author{
Tim Paulden and David K. Smith \\ School of Engineering, Computer Science and Mathematics \\ University of Exeter, UK \\ t.j.paulden@exeter.ac.uk / d.k.smith@exeter.ac.uk
}

Submitted: Mar 5, 2007; Accepted: Aug 3, 2007; Published: Aug 9, 2007

Mathematics Subject Classifications: 05C05, 05C85, 60C05, 68R05, 68R10, 68R15

\begin{abstract}
The Prüfer Code is a bijection between the $n^{n-2}$ trees on the vertex set $[1, n]$ and the $n^{n-2}$ strings in the set $[1, n]^{n-2}$ (known as Prüfer strings of order $n$ ). Efficient linear-time algorithms for decoding (i.e., converting string to tree) and encoding (i.e., converting tree to string) are well-known. In this paper, we examine an improved decoding algorithm (due to Cho et al.) that scans the elements of the Prüfer string in reverse order, rather than in the usual forward direction. We show that the algorithm runs in linear time without requiring additional data strutures or sorting routines, and is an 'online' algorithm - every time a new string element is read, the algorithm can correctly output an additional tree edge without any knowledge of the future composition of the string.

This new decoding algorithm allows us to derive results concerning the 'locality' properties of the Prüfer Code (i.e., the effect of making small changes to a Prüfer string on the structure of the corresponding tree). First, we show that mutating the $\mu$ th element of a Prüfer string (of any order) causes at most $\mu+1$ edge-changes in the corresponding tree. We also show that randomly mutating the first element of a random Prüfer string of order $n$ causes two edge-changes in the corresponding tree with probability $2(n-3) / n(n-1)$, and one edge-change otherwise. Then, based on computer-aided enumerations, we make three conjectures concerning the locality properties of the Prüfer Code, including a formula for the probability that a random mutation to the $\mu$ th element of a random Prüfer string of order $n$ causes exactly one edge-change in the corresponding tree. We show that if this formula is correct, then the probability that a random mutation to a random Prüfer string of order $n$ causes exactly one edge-change in the corresponding tree is asymptotically equal to one-third, as $n$ tends to infinity.
\end{abstract}




\section{Introduction}

\section{$1.1 \quad$ Background}

Let $\mathcal{T}_{n}$ denote the set of all possible free trees (i.e., connected acyclic graphs) on the vertex set $[1, n]=\{1,2, \ldots, n\}$. It is well-known that the number of trees in $\mathcal{T}_{n}$ is given by Cayley's celebrated formula $\left|\mathcal{T}_{n}\right|=n^{n-2}$, originally published in 1889 [3].

The first combinatorial proof of Cayley's formula was devised in 1918 by Prüfer [19], who constructed an explicit bijection between the trees in the set $\mathcal{T}_{n}$ and the strings in the set $\mathcal{P}_{n}=[1, n]^{n-2}$. This bijection - which is described in the next subsection - is known as the 'Prüfer Code', and the string that corresponds to a given tree under the Prüfer Code is known as the 'Prüfer string' for that tree.

The terms 'encoding' and 'decoding' are used to describe the two different directions of the Prüfer Code bijection. 'Encoding' refers to the process of constructing the Prüfer string corresponding to a given tree, and 'decoding' refers to the process of constructing the tree corresponding to a given Prüfer string.

\subsection{The Prüfer Code bijection}

In this subsection, we recall the traditional encoding and decoding algorithms for the Prüfer Code. These algorithms are very well-known, and are described in a number of papers, books, and dissertations (see [6], [9], [14], [18], [21], [22], [24], [26], and [27]).

\subsubsection{The Prüfer Code encoding algorithm (from tree to Prüfer string)}

To encode a tree as its corresponding Prüfer string, we iteratively delete the leaf vertex with the smallest label and write down its unique neighbour, until just a single edge remains. For example, the unique Prüfer string corresponding to the tree $T \in \mathcal{T}_{15}$ shown in Figure 1 below is $P=(12,6,15,15,6,6,3,11,1,11,1,3,15) \in \mathcal{P}_{15}$. (In this example, the vertex deletions occur in the following order: $2,4,5,7,8,9,6,10,12,13,11,1,3$.)

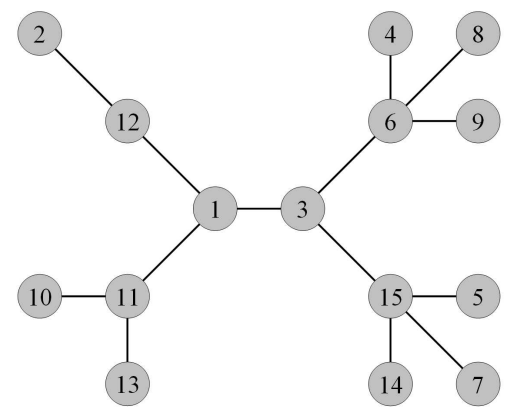

Figure 1: An example tree $T \in \mathcal{T}_{15}$. The unique Prüfer string corresponding to $T$ is $P=(12,6,15,15,6,6,3,11,1,11,1,3,15) \in \mathcal{P}_{15}$ 
Note that the degree of vertex $v$ in a tree is exactly one more than the number of times that $v$ occurs in the tree's Prüfer string. For instance, in the tree shown in Figure 1, the degree of vertex 1 is three, and there are two instances of the element 1 in the corresponding Prüfer string. This 'degree property' is well-known and easy to prove.

\subsubsection{The Prüfer Code decoding algorithm (from Prüfer string to tree)}

We now examine the traditional decoding algorithm for the Prüfer Code, which constructs the tree $T \in \mathcal{T}_{n}$ corresponding to a given Prüfer string $P=\left(p_{1}, p_{2}, \ldots, p_{n-2}\right) \in \mathcal{P}_{n}$.

In simple terms, the algorithm works by maintaining an 'eligible list' $L$ that specifies which vertices require exactly one more incident edge; this list makes it possible for the edges of the tree to be reconstructed from the Prüfer string in the same order as they were deleted during the encoding process.

The decoding algorithm operates as follows. First, the eligible list $L$ is initialised so that it contains all the elements of $[1, n]$ that do not occur in $P$. (These are precisely the leaf vertices in the tree $T$, due to the degree property noted above.) We then perform $n-2$ steps, indexed by $j=1,2, \ldots, n-2$. On step $j$, we perform the following three actions: (a) Create an edge between $p_{j}$ and the smallest element of $L$; (b) Delete from $L$ the smallest element of $L$; (c) Add the element $p_{j}$ to $L$ if this element does not occur again in $P$ (i.e., if $p_{j} \neq p_{j+t}$ for each $t \in[1, n-2-j]$ ). Once these $n-2$ steps have been completed, we then create an edge between the two remaining elements of $L$. The $n-1$ edges generated by this process form the tree $T$ corresponding to the Prüfer string $P$.

To illustrate this decoding procedure, suppose we reverse the example in the previous subsection, by decoding the Prüfer string $P=(12,6,15,15,6,6,3,11,1,11,1,3,15) \in \mathcal{P}_{15}$ into the corresponding tree $T \in \mathcal{T}_{15}$. Working through the steps of the decoding algorithm, we find that the fourteen edges produced are $(2,12),(4,6),(5,15),(7,15),(8,6),(9,6)$, $(6,3),(10,11),(12,1),(13,11),(11,1),(1,3),(3,15)$, and $(14,15)$. These are precisely the edges of the tree shown in Figure 1, and so the decoding algorithm has indeed reversed the encoding algorithm.

As noted earlier, the traditional decoding algorithm creates the edges of the tree in the same order as the encoding algorithm deletes these edges.

\section{A superior decoding algorithm for the Prüfer Code}

Naïve implementations of the Prüfer Code's encoding and decoding algorithms require $O\left(n^{2}\right)$ computational time, and as a consequence, many researchers have investigated alternative ways to implement these algorithms that are more computationally efficient.

It is well-known that intelligent use of data structures can reduce the computational time of the algorithms to $O(n \log n)$ [10]. Further research has resulted in decoding and encoding algorithms for the Prüfer Code that run in $O(n)$ time (see [1], [4], pp.663-665 of [7], [12], and pp. 270-273 of [13]); this is optimal complexity, since the length of each Prüfer string and the number of vertices in each tree are $O(n)$. 
However, all of these previous linear-time approaches are rather complicated, because they require one to preprocess the Prüfer string (in the case of decoding) or the tree (in the case of encoding). Furthermore, some of the approaches require the use of additional data structures or sorting routines. For instance, in the linear-time algorithms given by Caminiti et al. [1], one must extract certain structural information from the Prüfer string or tree, and then invoke an integer-sorting routine. Similarly, in the linear-time decoding algorithm devised by Klingsberg (see pp. 663-665 of [7], or pp. 270-273 of [13]), one must preprocess the Prüfer string, and then maintain two 'moving pointers' during decoding to identify the smallest available leaf at each stage.

In this section, we describe a novel decoding algorithm, known as 'Algorithm D', which is the simplest and most efficient method yet devised for converting a Prüfer string into its corresponding tree. We are not the first researchers to discover this algorithm - it originally appeared in [5], and also features in [8] and [23] — but we are the first to observe that it has $O(n)$ computational complexity and several other remarkable properties not possessed by any of the alternative Prüfer Code decoding algorithms.

\subsection{The structure of Algorithm D}

The following algorithm builds the tree $T \in \mathcal{T}_{n}$ corresponding to a Prüfer string $P \in \mathcal{P}_{n}$ by examining the string from right to left.

ALGORITHM D - A superior decoding algorithm for the Prüfer Code

Input - A Prüfer string $P=\left(p_{1}, p_{2}, \ldots, p_{n-2}\right) \in \mathcal{P}_{n}$, where $n \geq 3$.

Output - The tree $T \in \mathcal{T}_{n}$ corresponding to $P$ under the Prüfer Code bijection.

Step 1 - Let $T_{1}$ be the trivial subtree consisting of the vertex $n$, with no edges attached. Mark vertex $n$ as 'tight' (i.e., included in the current subtree), and vertices 1 to $n-1$ as 'loose' (i.e., not included in the current subtree). Define $p_{n-1}=n$. Let $j=2$.

Step 2 - If $p_{n-j}$ is loose, then let $v_{j}=p_{n-j}$. If $p_{n-j}$ is tight, then let $v_{j}$ be the largestlabelled loose vertex. (Note that $v_{j}$ is loose in either case.)

Step 3 - Form the next subtree $T_{j}$ by adding the vertex $v_{j}$ and the edge $\left(p_{n-j+1}, v_{j}\right)$ to the current subtree $T_{j-1}$, and change the status of $v_{j}$ from loose to tight.

Step 4 - Increment $j$ by one. If $j<n$, then go to Step 2; otherwise, proceed to Step 5.

Step 5 - Let $v_{n}$ be the one remaining loose vertex.

Step 6 - Form the final tree $T_{n}$ by adding the vertex $v_{n}$ and the edge $\left(p_{1}, v_{n}\right)$ to the current subtree $T_{n-1}$, and change the status of $v_{n}$ from loose to tight.

Step 7 - The required tree $T=T_{n}$ has been determined, so the algorithm terminates.

Note that the subtree $T_{j}$ consists of $j-1$ edges and $j$ vertices (namely, the $j$ tight vertices at that point), and the subtree $T_{j+1}$ is created by connecting an additional loose vertex to $T_{j}$ with an additional edge. The final tree $T_{n}$ produced by the algorithm is the required tree $T \in \mathcal{T}_{n}$ corresponding to the Prüfer string $P \in \mathcal{P}_{n}$. 


\subsection{An example of Algorithm D}

If the Prüfer string $P=(12,6,15,15,6,6,3,11,1,11,1,3,15) \in \mathcal{P}_{15}$ (which was introduced in Section 1.2) is the input to Algorithm $\mathrm{D}$, then the algorithm outputs the tree $T \in \mathcal{T}_{15}$ shown in Figure 1. The first seven subtrees produced during the algorithm are:

$T_{1}$ : Vertex $\{15\}$, no edges;

$T_{2}$ : Vertices $\{15,14\}$, edges $\{(15,14)\}$;

$T_{3}$ : Vertices $\{15,14,3\}$, edges $\{(15,14),(15,3)\}$;

$T_{4}$ : Vertices $\{15,14,3,1\}$, edges $\{(15,14),(15,3),(3,1)\}$;

$T_{5}$ : Vertices $\{15,14,3,1,11\}$, edges $\{(15,14),(15,3),(3,1),(1,11)\}$;

$T_{6}$ : Vertices $\{15,14,3,1,11,13\}$, edges $\{(15,14),(15,3),(3,1),(1,11),(11,13)\}$;

$T_{7}$ : Vertices $\{15,14,3,1,11,13,12\}$, edges $\{(15,14),(15,3),(3,1),(1,11),(11,13),(1,12)\}$.

Note that Algorithm D generates the $n-1$ edges of the tree $T$ in the opposite order to the traditional Prüfer Code decoding algorithm.

\subsection{Some remarks on Algorithm D}

\subsubsection{Optimal computational complexity}

It is straightforward to show that Algorithm $\mathrm{D}$ runs in $O(n)$ time. In implementing the algorithm, the most natural data structures to use would be a binary array to record the loose/tight status of each vertex, and an additional position variable, initialised to the value $n-1$, to scan this array. To determine the largest-labelled loose vertex (when this information is required in Step 2), or to determine the final loose vertex (in Step 5), we can simply decrement the position variable until a loose vertex is found. Since the variable position is decremented no more than $n-2$ times, it is obvious that the algorithm runs in $O(n)$ time overall.

An implementation of Algorithm D based around the data structures described above would appear to be optimally fast in terms of the total number of operations required to decode the Prüfer string $P$. However, if we wish to guarantee that the algorithm uses constant time per string element examined, we should instead maintain a doubly linked list containing the loose vertices in label order. (We recall that a 'doubly linked list' is a list in which each item has two pointers, one pointing to the previous item and one pointing to the next item.) Each time a loose vertex becomes tight, this vertex should be removed from the doubly linked list, and the pointers of its neighbours updated accordingly; this ensures that the largest-labelled loose vertex can be identified in a constant number of operations at any stage of the algorithm. It is easy to see that this alternative implementation also runs in $O(n)$ time overall.

Under either implementation described above, Algorithm D is likely to run noticeably faster than existing $O(n)$ decoding algorithms for the Prüfer Code, as it is extremely parsimonious in its use of data structures, and does not require the Prüfer string to undergo any form of preprocessing. 


\subsubsection{Algorithm D is an online algorithm}

It is also worth noting that Algorithm $\mathrm{D}$ is an 'online algorithm'. As the string $P$ is read from right to left, the algorithm correctly outputs an additional edge of the corresponding tree $T$ every time a new string element is read, without any knowledge about the 'unseen' portion of the string. Thus, for any $k \in[1, n-3]$, the algorithm is able to output $k$ edges of $T$ based only on the $k$ rightmost elements of $P$ - and when the algorithm finally reads the leftmost element of $P$, it is able to output the final two edges of $T$.

To illustrate this point, consider the Prüfer string $P=(7,4,1,5,3,5) \in \mathcal{P}_{8}$. When this Prüfer string is fed into Algorithm $\mathrm{D}$, the seven edges of the corresponding tree $T \in \mathcal{T}_{8}$ are generated in the following order: $(8,5),(5,3),(3,7),(5,1),(1,4),(4,6)$, and $(7,2)$. Clearly, in determining the first three of these seven edges - namely, $(8,5),(5,3)$, and $(3,7)$ - Algorithm D only makes use of the last three string elements, $(\ldots, 5,3,5)$.

Interestingly, no algorithm that reads the Prüfer string from left to right can correctly output one new tree edge every time a new string element is read, in the manner of Algorithm D. To see that this is an impossible task, consider for example the Prüfer strings $P=(7,4,1,5,3,5) \in \mathcal{P}_{8}$ and $P^{\prime}=(7,4,8,5,2,6) \in \mathcal{P}_{8}$. Although these strings match in both position one and position two, their corresponding trees have no edges in common. Therefore, the fact that a string in $\mathcal{P}_{8}$ begins $(7,4, \ldots)$ provides insufficient information to determine any edge of the corresponding tree with certainty, and so a left-to-right decoding algorithm can never exhibit the online character of Algorithm D.

\subsubsection{The 'nested' nature of Prüfer strings}

The online property of Algorithm D described in the previous subsection relies on the fact that the Prüfer Code correspondence between trees and Prüfer strings possesses a distinctive 'nested' structure - but only if we consider the Prüfer string elements in right-to-left order. Specifically, if two Prüfer strings end with the same $k$ elements, then their corresponding trees have at least $k$ common edges.

For example, consider the Prüfer strings in $\mathcal{P}_{8}$. From the structure of Algorithm D, we see that any Prüfer string $P \in \mathcal{P}_{8}$ that ends with $(\ldots, 5)$ corresponds to a tree containing the edge $(8,5)$. Extending this reasoning further, any Prüfer string $P \in \mathcal{P}_{8}$ that ends with $(\ldots, 3,5)$ corresponds to a tree containing the edges $(8,5)$ and $(5,3)$; any Prüfer string $P \in \mathcal{P}_{8}$ that ends with $(\ldots, 5,3,5)$ corresponds to a tree containing the edges $(8,5),(5,3)$, and $(3,7)$; and so on. Thus, if two Prüfer strings agree in their last three positions, such as $P=(7,4,1,5,3,5) \in \mathcal{P}_{8}$ and $P^{\prime \prime}=(7,4,5,5,3,5) \in \mathcal{P}_{8}$, then their corresponding trees $T$ and $T^{\prime \prime}$ must have at least three common edges. (In this example, it is easy to show that $T$ and $T^{\prime \prime}$ have no other common edges, but this will not always be the case.)

The nesting property described above could be valuable in a practical context, since the Prüfer Code has already been deployed for indexing applications, such as PRIX [20].

Finally, we note that no similar nesting structure exists if the string elements are considered in the usual left-to-right direction. This fact is illustrated by the Prüfer strings $P=(7,4,1,5,3,5) \in \mathcal{P}_{8}$ and $P^{\prime}=(7,4,8,5,2,6) \in \mathcal{P}_{8}$ introduced earlier - these strings match in their first two elements, but their corresponding trees have no common edges. 


\subsubsection{The analytical importance of Algorithm D}

It is much easier to analyse the properties of the Prüfer Code using Algorithm D, compared to alternative decoding algorithms for the Prüfer Code. This is because Algorithm D does not require one to preprocess the string in any way, or look ahead to determine whether or not elements occur again 'later' in the string. Consequently, Algorithm D allows us to prove a number of results concerning the Prüfer Code that are exceedingly complex to prove (or even intractable) using other decoding algorithms. Indeed, some of the results derived in [8] and [23] rely crucially on the structure of the new decoding algorithm.

\section{Basic locality results for the Prüfer Code}

\subsection{Introduction to locality}

The locality of a tree representation such as the Prüfer Code is a measure of the regularity of the mapping between the tree space and the string space (i.e., $\mathcal{T}_{n}$ and $\mathcal{P}_{n}$, in the case of the Prüfer Code). A tree representation has high locality if small changes to the string typically cause small changes to the corresponding tree, and low locality otherwise. The concept of locality is crucial in the field of genetic and evolutionary algorithms (GEAs), where research has indicated that it is highly desirable for a tree representation to possess high locality — for further details and related work in this area, see [2], [9], [14], [15], [16], [17], [21], [22], [24], and [25].

We quantify locality by examining the effect of mutating a single Prüfer string element on the structure of the corresponding tree. More formally, let $P \in \mathcal{P}_{n}$ be the original Prüfer string, and let $P^{\star} \in \mathcal{P}_{n}$ be the Prüfer string formed by mutating the $\mu$ th element of $P$ (thus, $p_{\mu} \neq p_{\mu}^{\star}$, and $p_{i}=p_{i}^{\star}$ for each $i \in[1, n-2] \backslash\{\mu\}$ ). If the trees corresponding to $P$ and $P^{\star}$ under the Prüfer Code are $T$ and $T^{\star}$, then the key measure of interest is the tree distance $\Delta \in[1, n-1]$ between the trees $T$ and $T^{\star}$ (i.e., the number of edge-changes required to transform one tree into the other.) Formally, $\Delta=n-1-\left|E(T) \cap E\left(T^{\star}\right)\right|$, where $E(T)$ and $E\left(T^{\star}\right)$ are the edge-sets of $T$ and $T^{\star}$.

(In this paper, we wish to measure the distance between trees with undirected edges; thus, $\Delta$ is a natural metric to use. For trees with directed edges, it would be natural to use a metric that regards the directed edges $(i \rightarrow j)$ and $(j \rightarrow i)$ as being distinct.)

Suppose that $n \geq 3$ and $\mu \in[1, n-2]$ are given. Since there are $n^{n-2}$ choices for the original Prüfer string $P \in \mathcal{P}_{n}$ and $n-1$ choices for the value of $p_{\mu}^{\star} \in[1, n] \backslash\left\{p_{\mu}\right\}$, the space of possible mutation events, $\mathcal{M}$, has cardinality $n^{n-2}(n-1)$. Each of these $n^{n-2}(n-1)$ mutation events has an associated value of $\Delta$, and the locality of the Prüfer Code is characterised by the distribution of $\Delta$ over the space $\mathcal{M}$. High-locality mutation events have small values of $\Delta$ associated with them, and low-locality mutation events have large values of $\Delta$ associated with them. A mutation event for which $\Delta=1$ (the smallest possible value of $\Delta$ ) is known as 'perfect' or 'optimal'.

In this remainder of this section, we develop some basic locality results concerning the Prüfer Code; these results are then extended and generalised in Section 4. 


\subsection{A simple bound on $\Delta$}

The following theorem shows that mutating the $\mu$ th element of a Prüfer string can never cause more than $\mu+1$ edge-changes in the corresponding tree. This theorem was first established in 2003 by Thompson (see [24], pp. 190-193) but the proof required several pages of intricate analysis; using Algorithm D, the proof is almost immediate.

Theorem 1. For any Prüfer string $P=\left(p_{1}, p_{2}, \ldots, p_{n-2}\right) \in \mathcal{P}_{n}$, altering the value of the element $p_{\mu}$ (whilst leaving the other $n-3$ elements of $P$ unchanged) changes at most $\mu+1$ edges of the corresponding tree, for any $\mu \in[1, n-2]$.

Proof. Let $P$ and $P^{\star}$ be two Prüfer strings that differ only in element $\mu$ (thus, $p_{\mu} \neq p_{\mu}^{\star}$, and $p_{i}=p_{i}^{\star}$ for each $\left.i \in[1, n-2] \backslash\{\mu\}\right)$. Since $P$ and $P^{\star}$ match in their last $n-2-\mu$ elements, the subtree $T_{n-1-\mu}$ formed during the execution of Algorithm $\mathrm{D}$ is the same when the input string is $P$ as when the input string is $P^{\star}$. It follows that the trees corresponding to $P$ and $P^{\star}$ must have at least $n-2-\mu$ common edges - that is, they differ in no more than $\mu+1$ edges.

It is easy to show that, for any $n \geq 5$ and any $\mu \in[1, n-2]$, the distribution of $\Delta$ extends all the way to $\Delta=\mu+1$ (i.e., mutation events exist that give rise to $\mu+1$ edge-changes):

- If $\mu=1$, consider the mutation event for which $P=(3, n, n, \ldots, n)$ and the new first element is $p_{1}^{\star}=1$;

- If $\mu=2$, consider the mutation event for which $P=(n-1,3, n, n, \ldots, n)$ and the new second element is $p_{2}^{\star}=1$;

- If $\mu \geq 3$, consider the mutation event for which $P=(3,4, \ldots, n)$ and the new $\mu$ th element is $p_{\mu}^{\star}=1$.

Therefore, for each $\mu \in[1, n-2]$, the bound $\Delta \leq \mu+1$ that is specified by Theorem 1 is as tight as possible.

It is worth commenting briefly on the existence of analogous results for alternative tree representations. For instance, it is shown in [17] that a similar result to Theorem 1 holds for the 'Blob Code' tree representation - specifically, mutating the $\mu$ th element of a 'Blob string' causes at most $n-\mu$ edge-changes in the corresponding tree. An even stronger result holds for the 'Dandelion Code' tree representation - a single-element mutation to a 'Dandelion string' can never cause more than five edge-changes in the corresponding tree, for any value of $n$ [15], [25]. For further analysis and results relating to these alternative representations, the reader is referred to [2], [11], [12], [15], [16], [17], [18], and [25].

\subsection{The distribution of $\Delta$ when $\mu=1$}

In this subsection, we focus on the case $\mu=1$ (i.e., mutating the leftmost element of the Prüfer string). In this case, Theorem 1 tells us that the tree distance $\Delta$ between the 
trees corresponding to $P$ and $P^{\star}$ must be either 1 or 2 . In this subsection, we analyse the circumstances under which each of these values can arise.

First, for ease of exposition, we define some additional notation. Since $P$ and $P^{\star}$ match in their last $n-3$ elements, the subtree $T_{n-2}$ formed during the execution of Algorithm $\mathrm{D}$ is the same when the input string is $P$ as when the input string is $P^{\star}$. Let $x_{1}$ and $x_{2}$ be the two vertices in $[1, n]$ not belonging to $T_{n-2}$ (where $x_{1}<x_{2}$ ), let $y$ be equal to $p_{2}$ if $n>3$ (and equal to 3 if $n=3$ ), and let $Z$ be the set containing all vertices in the subtree $T_{n-2}$ other than $y$. Therefore, $|Z|=n-3$, and $\left\{x_{1}, x_{2}, y\right\} \cup Z=[1, n]$.

Now observe that the tree $T$ corresponding to $P$ is created by adding two further edges to the subtree $T_{n-2}$, following the rules of the decoding algorithm. Exactly which two edges are added depends only on the value of $p_{1}$, as follows:

- If $p_{1}=x_{1}$, then the added edges are $\left(y, x_{1}\right)$ and $\left(x_{1}, x_{2}\right)$;

- If $p_{1}=x_{2}$, then the added edges are $\left(y, x_{2}\right)$ and $\left(x_{2}, x_{1}\right)$;

- If $p_{1}=y$, then the added edges are $\left(y, x_{2}\right)$ and $\left(y, x_{1}\right)$;

- If $p_{1}=z$, where $z$ is any value in $Z$, then the added edges are $\left(y, x_{2}\right)$ and $\left(z, x_{1}\right)$.

Of course, exactly the same reasoning holds for the string $P^{\star}$, except that $p_{1}^{\star}$ takes the place of $p_{1}$ in each of the four cases described above.

It is then easy to confirm that the tree distance between $T$ and $T^{\star}$ will be equal to 2 only in two circumstances: (i) if $p_{1}=x_{1}$ and $p_{1}^{\star}=z \in Z$; (ii) if $p_{1}=z \in Z$ and $p_{1}^{\star}=x_{1}$. In all other cases, the tree distance will be equal to 1 .

We now reformulate this finding in probabilistic terms. Suppose that $P$ is a Prüfer string generated uniformly at random from $\mathcal{P}_{n}$, and $P^{\star}$ is the Prüfer string produced when the value of $p_{1}$ is randomly mutated to some new value $p_{1}^{\star} \in[1, n] \backslash\left\{p_{1}\right\}$ (with all $n-1$ alternative values being equally likely). Under this scenario, the probability of case (i) arising (i.e., the probability that $p_{1}$ is equal to $x_{1}$ and $p_{1}^{\star}$ belongs to $Z$ ) is clearly $(n-3) / n(n-1)$, and the probability of case (ii) arising (i.e., the probability that $p_{1}$ belongs to $Z$ and $p_{1}^{\star}$ is equal to $\left.x_{1}\right)$ is also $(n-3) / n(n-1)$.

We have therefore proved the following theorem.

Theorem 2. The probability that a random mutation to the first element of a random Prüfer string $P \in \mathcal{P}_{n}$ causes two edge-changes in the corresponding tree is

$$
\mathbb{P}(\Delta=2 \mid \mu=1)=\frac{2(n-3)}{n(n-1)},
$$

and the probability that this mutation causes one edge-change in the corresponding tree is

$$
\mathbb{P}(\Delta=1 \mid \mu=1)=1-\frac{2(n-3)}{n(n-1)} .
$$

Once again, this result was proved by Thompson (see [24], pp. 196-202), but the proof required many pages of reasoning. Using Algorithm D, the proof is significantly shorter. 


\section{Further locality results for the Prüfer Code}

Theorem 2 completely characterises the distribution of $\Delta$ under the Prüfer Code when the mutation position $\mu$ is equal to one. In this section, we extend this work by examining the distribution of $\Delta$ for larger values of $\mu$ using computer-aided enumerations.

We begin by introducing two additional pieces of notation that will be used in this section: firstly, the $\{X, y, Z\}$ partition of $[1, n]$; secondly, the $\left\{\mathcal{M}_{S}\right\}$ partition of $\mathcal{M}$.

\subsection{Additional notation}

\subsubsection{The $\{X, y, Z\}$ partition of $[1, n]$}

Our first piece of additional notation is motivated by the usefulness of the partition $\left(x_{1}, x_{2}, y, Z\right)$ in the analysis of the case $\mu=1$.

Let $P$ be a Prüfer string generated uniformly at random from $\mathcal{P}_{n}$, and let $P^{\star}$ be the Prüfer string produced when the value of $p_{\mu}$ is randomly mutated to some new value $p_{\mu}^{\star} \in[1, n] \backslash\left\{p_{\mu}\right\}$ (with all $n-1$ alternative values being equally likely). When the strings $P$ and $P^{\star}$ are fed into Algorithm $\mathrm{D}$, the same subtree $T_{n-1-\mu}$ arises after $n-2-\mu$ edges have been created, as $P$ and $P^{\star}$ match in their last $n-2-\mu$ elements. Let $x_{1}, x_{2}, \ldots, x_{\mu+1}$ be the $\mu+1$ vertices in $[1, n]$ not belonging to the subtree $T_{n-1-\mu}\left(\right.$ where $\left.x_{1}<x_{2}<\ldots<x_{\mu+1}\right)$, and define $X=\left\{x_{1}, x_{2}, \ldots, x_{\mu+1}\right\}$. Let $y$ be equal to $p_{\mu+1}$ if $\mu \in[1, n-3]$, and equal to $n$ if $\mu=n-2$. Finally, let $Z$ be the set containing all vertices in the subtree $T_{n-1-\mu}$ other than $y$, and let the elements of $Z$ be denoted $z_{1}, z_{2}, \ldots, z_{n-2-\mu}$, where $z_{1}<z_{2}<\ldots<z_{n-2-\mu}$. Therefore, $|X|=\mu+1,|Z|=n-2-\mu$, and $X \cup\{y\} \cup Z=[1, n]$.

To illustrate the notation introduced above, we consider a simple example for $n=8$ and $\mu=3$. If the original Prüfer string is $P=(7,4,1,5,3,5)$ and the mutated Prüfer string is $P^{\star}=(7,4,4,5,3,5)$, then the subtree $T_{4}$ formed when either string is decoded using Algorithm D consists of the vertices $\{8,5,3,7\}$ and the edges $\{(8,5),(5,3),(3,7)\}$. Thus, $X=\left\{x_{1}, x_{2}, x_{3}, x_{4}\right\}=\{1,2,4,6\}, y=5$, and $Z=\left\{z_{1}, z_{2}, z_{3}\right\}=\{3,7,8\}$.

\subsubsection{The $\left\{\mathcal{M}_{S}\right\}$ partition of $\mathcal{M}$}

Our second piece of additional notation represents a natural partition of the mutation space $\mathcal{M}$ defined earlier.

For fixed $n \geq 3$ and fixed $\mu \in[1, n-2]$, recall that $\mathcal{M}$ is the space of all $n^{n-2}(n-1)$ Prüfer string mutation events in which the mutation position is $\mu$ (where each mutation event $M=\left(P, p_{\mu}^{\star}\right) \in \mathcal{M}$ represents a certain choice of the original Prüfer string $P \in \mathcal{P}_{n}$ and the new $\mu$ th element $\left.p_{\mu}^{\star}\right)$.

Now, we define $\mathcal{M}_{S}$ to be the subspace of $\mathcal{M}$ containing all mutation events for which

the associated Prüfer string $P$ ends with the substring $S \in[1, n]^{n-2-\mu}$ (i.e., the rightmost $n-2-\mu$ elements of $P$ coincide exactly with the string $S$ ).

Clearly, the $n^{n-2-\mu}$ subspaces $\left\{\mathcal{M}_{S}\right\}$ constitute a partition of $\mathcal{M}$, and each subspace contains $n^{\mu}(n-1)$ mutation events. 


\subsection{An important combinatorial result}

In this subsection, we establish a combinatorial result - Theorem 3 - that brings about major simplifications in our subsequent computer-aided analysis. The theorem shows that one may determine the distribution of $\Delta$ on the full mutation space $\mathcal{M}$ (which contains $n^{n-2}(n-1)$ mutation events) by calculating the distribution of $\Delta$ on one of the subspaces $\left\{M_{S}\right\}$ (which contains $n^{\mu}(n-1)$ mutation events), and then scaling up this distribution by a factor of $n^{n-2-\mu}$. This observation significantly reduces the computational effort required to determine the distribution of $\Delta$ on $\mathcal{M}$, and therefore allows extensive numerical results to be obtained in a relatively short period of time.

Theorem 3. Under the definitions introduced above, the distribution of $\Delta$ on the subspace $\mathcal{M}_{S}$ is independent of the choice of $S \in[1, n]^{n-2-\mu}$.

Proof. It is enough to show that, for each $S \in[1, n]^{n-2-\mu}$, the distribution of $\Delta$ on $\mathcal{M}_{S}$ is identical to the distribution of $\Delta$ on $\mathcal{M}_{\bar{S}}$, where $\bar{S}=(n, n, \ldots, n) \in[1, n]^{n-2-\mu}$. To do this, we exhibit an explicit $\Delta$-preserving bijection between $\mathcal{M}_{S}$ and $\mathcal{M}_{\bar{S}}$.

Given any string $S \in[1, n]^{n-2-\mu}$, observe that each mutation event in the subspace $\mathcal{M}_{S}$ has the same associated variables $X=\left\{x_{1}, x_{2}, \ldots, x_{\mu+1}\right\}, y$, and $Z=\left\{z_{1}, z_{2}, \ldots, z_{n-2-\mu}\right\}$, as defined in subsection 4.1. (To see this, note that any mutation event $M=\left(P, p^{\star}\right) \in \mathcal{M}_{S}$ defines two Prüfer strings $P$ and $P^{\star}$, both of which end with $S$.) Given these variables, we may define a permutation $\phi_{S}:[1, n] \rightarrow[1, n]$ such that $\phi_{S}\left(x_{i}\right)=i$ for each $i \in[1, \mu+1]$, $\phi_{S}\left(z_{i}\right)=\mu+1+i$ for each $i \in[1, n-2-\mu]$, and $\phi_{S}(y)=n$. (This definition is motivated by the fact that the $\{X, y, Z\}$ variables associated with any mutation event $M \in \mathcal{M}_{\bar{S}}$ are $X=\{1,2, \ldots, \mu+1\}, y=n$, and $Z=\{\mu+2, \mu+3, \ldots, n-1\}$.

We now demonstrate that an arbitrarily-chosen mutation event $M=\left(P, p_{\mu}^{\star}\right) \in \mathcal{M}_{S}$ gives rise to exactly the same value of $\Delta$ as the mutation event $\bar{M}=\left(\bar{P}, \overline{p_{\mu}^{\star}}\right) \in \mathcal{M}_{\bar{S}}$, where $\bar{P}=\left(\phi_{S}\left(p_{1}\right), \phi_{S}\left(p_{2}\right), \ldots, \phi_{S}\left(p_{\mu}\right), n, n, \ldots, n\right)$ and $\overline{p_{\mu}^{\star}}=\phi_{S}\left(p_{\mu}^{\star}\right)$.

Following the notation introduced earlier in the paper, let the Prüfer strings associated with $M$ be $P$ and $P^{\star}$, and let their corresponding trees be $T$ and $T^{\star}$ respectively. Similarly, let the Prüfer strings associated with $\bar{M}$ be $\bar{P}$ and $\bar{P}^{\star}$, and let their corresponding trees be $\bar{T}$ and $\bar{T}^{\star}$ respectively. As noted earlier, when $P$ and $P^{\star}$ are fed into Algorithm $\mathrm{D}$, the same subtree $T_{n-1-\mu}$ arises after $n-2-\mu$ edges have been created, and each of the trees $T$ and $T^{\star}$ is created by adding $\mu+1$ edges to $T_{n-1-\mu}$. Similarly, when $\bar{P}$ and $\bar{P}^{\star}$ are fed into Algorithm D, the same subtree $\bar{T}_{n-1-\mu}$ arises after $n-2-\mu$ edges have been created, and each of the trees $\bar{T}$ and $\bar{T}^{\star}$ is created by adding $\mu+1$ edges to $\bar{T}_{n-1-\mu}$.

We now make an important observation: if $(i, j)$ is the $k$ th edge added to $T_{n-1-\mu}$ to form $T$ (where $k \in[1, \mu+1]$ ), then $\left(\phi_{S}(i), \phi_{S}(j)\right)$ is the $k$ th edge added to $\bar{T}_{n-1-\mu}$ to form $\bar{T}$; similarly, if $(i, j)$ is the $k$ th edge added to $T_{n-1-\mu}$ to form $T^{\star}$ (where $k \in[1, \mu+1]$ ), then $\left(\phi_{S}(i), \phi_{S}(j)\right)$ is the $k$ th edge added to $\bar{T}_{n-1-\mu}$ to form $\bar{T}^{\star}$. (A simple example that illustrates this property is given in Remark 1 below.) Since $\phi_{S}$ is a permutation, it follows that the number of edges common to the trees $T$ and $T^{\star}$ is the same as the number of edges common to the trees $\bar{T}$ and $\bar{T}^{\star}$.

This analysis demonstrates that $M$ and $\bar{M}$ always give rise to the same value of $\Delta$. Therefore, if we associate each $M \in \mathcal{M}_{S}$ with its corresponding $\bar{M} \in \mathcal{M}_{\bar{S}}$, we obtain a 
$\Delta$-preserving bijection between $\mathcal{M}_{S}$ and $\mathcal{M}_{\bar{S}}$. It follows that the distribution of $\Delta$ on $\mathcal{M}_{S}$ is identical to the distribution of $\Delta$ on $\mathcal{M}_{\bar{S}}$, as required.

Remark 1. The following example may help to make the structure of the $\Delta$-preserving bijection a little more transparent. Suppose that $n=8$ and $\mu=3$. In this case, there are $8^{3}$ subspaces $\left\{\mathcal{M}_{S}\right\}$, indexed by the strings in $[1,8]^{3}$, with each subspace containing $8^{3} \times 7=3,584$ mutation events. Since $n=8$, the string $\bar{S}$ is $(8,8,8)$, and the $\{X, y, Z\}$ variables associated with any mutation event $M \in \mathcal{M}_{\bar{S}}$ are therefore $X=\{1,2,3,4\}$, $y=8$, and $Z=\{5,6,7\}$.

Now consider the string $S=(5,3,5)$, and the associated subspace $\mathcal{M}_{(5,3,5)}$. For any mutation event $M \in \mathcal{M}_{(5,3,5)}$, the associated variables $\{X, y, Z\}$ are $X=\{1,2,4,6\}$, $y=5$, and $Z=\{3,7,8\}$. Therefore, the associated permutation $\phi_{(5,3,5)}$ maps $[1,8]$ to $[1,8]$ as follows: $1 \mapsto 1,2 \mapsto 2,4 \mapsto 3,6 \mapsto 4,3 \mapsto 5,7 \mapsto 6,8 \mapsto 7$, and $5 \mapsto 8$. Thus, under the correspondence described in the third paragraph of the above proof, the mutation event $M=((7,4,1,5,3,5), 4) \in \mathcal{M}_{(5,3,5)}$ is mapped to the mutation event $\bar{M}=((6,3,1,8,8,8), 3) \in \mathcal{M}_{(8,8,8)}$.

Let us now consider the structure of the trees associated with the mutation events $M$ and $\bar{M}$ described above. It is easy to see that the mutation event $M$ represents the Prüfer strings $P=(7,4,1,5,3,5)$ and $P^{\star}=(7,4,4,5,3,5)$, and the corresponding trees $T$ and $T^{\star}$ are each created by adding four more edges to the common subtree $T_{4}$ (which has vertices $\{8,5,3,7\}$ and edges $\{(8,5),(5,3),(3,7)\})$. The tree $T$ is formed by adding $(5,1),(1,4),(4,6)$, and $(7,2)$ to $T_{4}$; the tree $T^{\star}$ is formed by adding $(5,4),(4,6),(4,2)$, and $(7,1)$ to $T_{4}$. Clearly, the value of $\Delta$ associated with $M$ is three.

Similarly, the mutation event $\bar{M}$ represents the Prüfer strings $P=(6,3,1,8,8,8)$ and $P^{\star}=(6,3,3,8,8,8)$, and the corresponding trees $\bar{T}$ and $\bar{T}^{\star}$ are each created by adding four more edges to the common subtree $\bar{T}_{4}$ (which has vertices $\{8,7,6,5\}$ and edges $\{(8,7),(8,6),(8,5)\})$. The tree $\bar{T}$ is formed by adding $(8,1),(1,3),(3,4)$, and $(6,2)$ to $\bar{T}_{4}$; the tree $\bar{T}^{\star}$ is formed by adding $(8,3),(3,4),(3,2)$, and $(6,1)$ to $\bar{T}_{4}$. We see that the value of $\Delta$ associated with $\bar{M}$ is three - therefore, $M$ and $\bar{M}$ do indeed give rise to the same value of $\Delta$.

Finally, we observe that the edge sets described in the last two paragraphs confirm the interrelationships identified in the proof of Theorem 3: if $(i, j)$ is the $k$ th edge added to $T_{4}$ to form $T$, then $\left(\phi_{S}(i), \phi_{S}(j)\right)$ is the $k$ th edge added to $\bar{T}_{4}$ to form $\bar{T}$; similarly, if $(i, j)$ is the $k$ th edge added to $T_{4}$ to form $T^{\star}$, then $\left(\phi_{S}(i), \phi_{S}(j)\right)$ is the $k$ th edge added to $\bar{T}_{4}$ to form $\bar{T}^{\star}$. For instance, the third edge added to $T_{4}$ to form $T$ is $(4,6)$, and the third edge added to $\bar{T}_{4}$ to form $\bar{T}$ is $(3,4)=\left(\phi_{S}(4), \phi_{S}(6)\right)$. As noted earlier, these interrelationships guarantee that $M$ and $\bar{M}$ always have the same associated value of $\Delta$.

\subsection{Computer-aided enumeration results}

For any $n \geq 3$ and any mutation position $\mu \in[1, n-2]$, suppose we wish to investigate the locality of the Prüfer Code by determining the distribution of $\Delta$ over the $n^{n-2}(n-1)$ mutation events in the space $\mathcal{M}$. Theorem 3 reveals that this distribution may be obtained by scaling-up the distribution of $\Delta$ on the subspace $\mathcal{M}_{\bar{S}}$ by a factor of $n^{n-2-\mu}$. Therefore, 
by exploiting Theorem 3 , we only need to examine the $n^{\mu}(n-1)$ mutation events in $\mathcal{M}_{\bar{S}}$, rather than the $n^{n-2}(n-1)$ mutation events in $\mathcal{M}$.

We now define $N(\mu, n, \Delta)$ to be the number of mutation events in $\mathcal{M}_{\bar{S}}$ in which precisely $\Delta$ edge-changes take place (where $\Delta \in[1, \mu+1]$, by Theorem 1 ). Through exhaustive enumerations, aided by a computer, the value of $N(\mu, n, \Delta)$ was determined exactly for each $\mu \in[1,7]$ and each $n \in[\mu+2, \mu+11]$. (Note that, for any given value of $\mu \geq 1$, the smallest valid value of $n$ is $\mu+2$.)

Analysis of the resulting figures using a computer algebra system showed that, for fixed $\mu$ and $\Delta$, the values of $N(\mu, n, \Delta)$ that were computed followed simple polynomials in $n$. (We were motivated to seek polynomial relationships in the values of $N(\mu, n, \Delta)$ by the fact that $N(1, n, 1)=n^{2}-3 n+6$ and $N(1, n, 2)=2 n-6$, as shown in the analysis preceding Theorem 2.) The fitted polynomials gave the exact value of $N(\mu, n, \Delta)$ for every value of $n$ considered, and in every case, the number of $n$-values examined was at least two more than the degree of the polynomial (thus precluding the possibility of the polynomials simply overfitting the data). Furthermore, for each value of $\mu$, the $\mu+1$ associated polynomials were found to total exactly $n^{\mu}(n-1)$.

Our results are summarised on the following pages. For each $\mu \in[1,3]$, we tabulate the values of $N(\mu, n, \Delta)$ that were computed, and identify the polynomials that describe the values in each row. For each $\mu \in[4,7]$, we just identify the polynomials, to conserve space; the corresponding tables can easily be reconstructed from these polynomials.

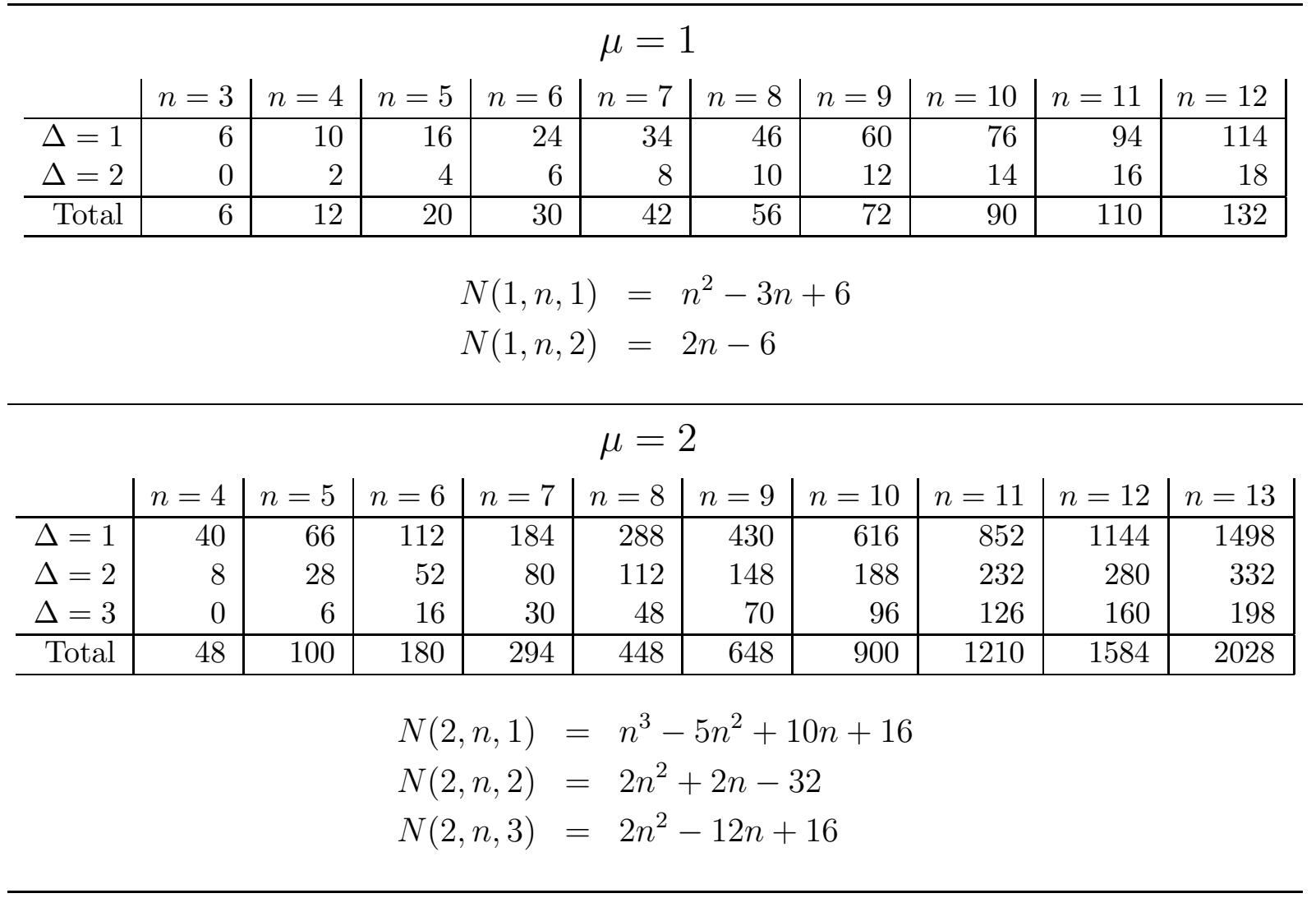




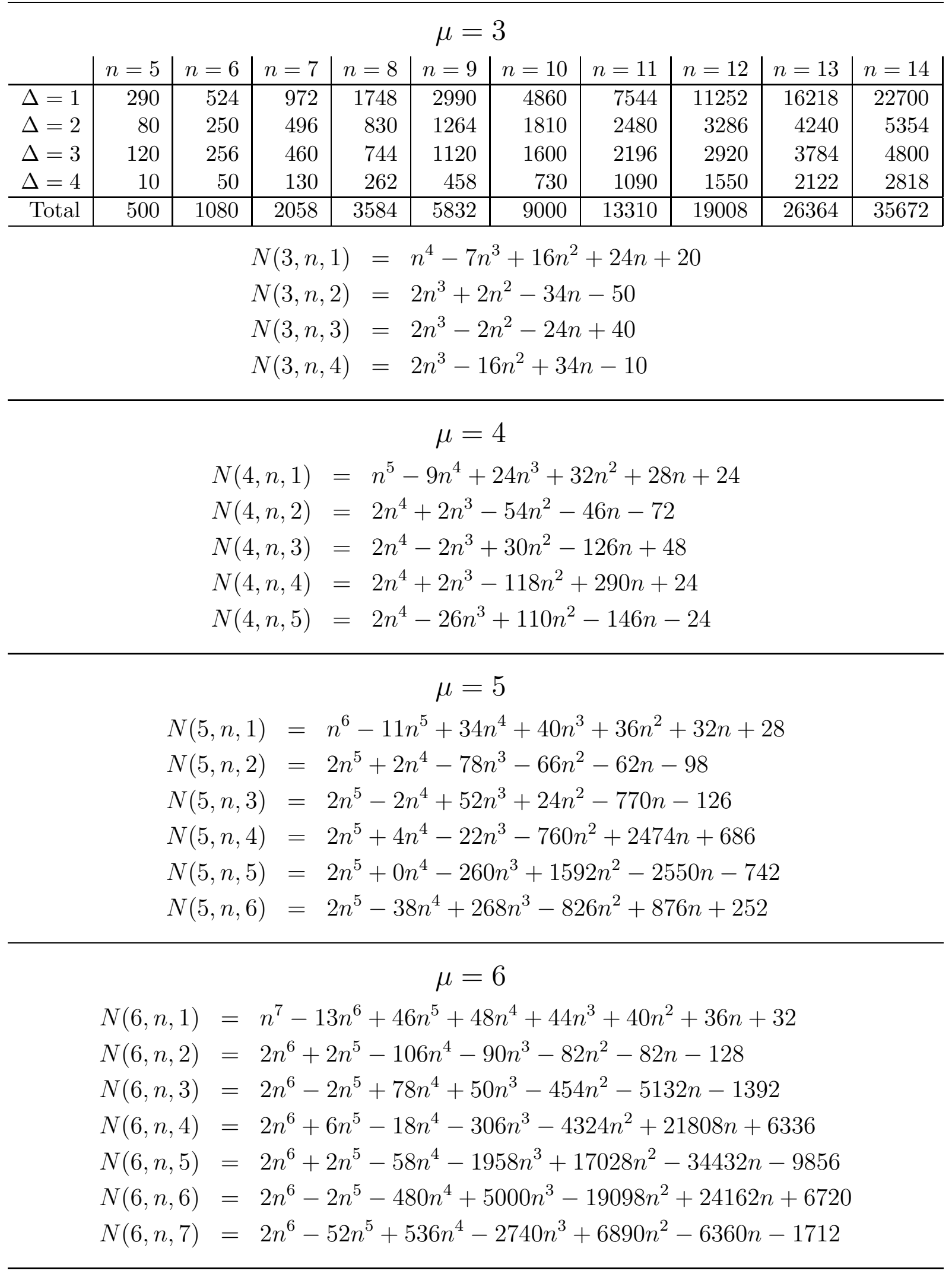




$$
\begin{gathered}
\mu=7 \\
N(7, n, 1)=n^{8}-15 n^{7}+60 n^{6}+56 n^{5}+52 n^{4}+48 n^{3}+44 n^{2}+40 n+36 \\
N(7, n, 2)=2 n^{7}+2 n^{6}-138 n^{5}-118 n^{4}-106 n^{3}-102 n^{2}-106 n-162 \\
N(7, n, 3)=2 n^{7}-2 n^{6}+108 n^{5}+80 n^{4}-772 n^{3}-5672 n^{2}-37388 n-9360 \\
N(7, n, 4)=2 n^{7}+8 n^{6}-12 n^{5}-392 n^{4}-1818 n^{3}-24024 n^{2}+201146 n+50364 \\
N(7, n, 5)=2 n^{7}+4 n^{6}-60 n^{5}-440 n^{4}-14134 n^{3}+169350 n^{2}-431452 n-105876 \\
N(7, n, 6)=2 n^{7}+0 n^{6}-88 n^{5}-4644 n^{4}+66324 n^{3}-307140 n^{2}+462110 n+110718 \\
N(7, n, 7)=2 n^{7}-4 n^{6}-820 n^{5}+12540 n^{4}-78814 n^{3}+230990 n^{2}-247152 n-57744 \\
N(7, n, 8)=2 n^{7}-68 n^{6}+954 n^{5}-7078 n^{4}+29272 n^{3}-63446 n^{2}+52802 n+12024
\end{gathered}
$$

The results above show that certain polynomials (of relatively low degree) describe the values taken by $N(\mu, n, \Delta)$ as $n$ varies, so long as $\mu \in[1,7]$ and $n \in[\mu+2, \mu+11]$. The authors strongly believe that these polynomials are in fact valid for all values of $n$, and that larger values of $\mu$ would simply give rise to higher-order polynomial relationships (see Conjectures 1 to 3 in the following subsection).

It should also be noted that the expressions for $\mathbb{P}(\Delta=1 \mid \mu=1)$ and $\mathbb{P}(\Delta=2 \mid \mu=1)$ in Theorem 2 are obtained by dividing the expressions for $N(1, n, 1)$ and $N(1, n, 2)$ by $\left|\mathcal{M}_{\bar{S}}\right|=n(n-1)$. In light of Theorem 3, it is apparent that this is just a special case of the general result that $\mathbb{P}(\Delta \mid \mu)=N(\mu, n, \Delta) /\left(n^{\mu}(n-1)\right)$.

\subsection{Three new conjectures}

In this subsection, we make three conjectures relating to the enumeration results given above. Firstly, we propose a simple closed form expression for $N(\mu, n, 1)$. Observe that the values of $N(\mu, n, 1)$ for $\mu \in[1,7]$ are specified by the following polynomials:

$$
\begin{gathered}
N(1, n, 1)=n^{2}-3 n+6, \\
N(2, n, 1)=n^{3}-5 n^{2}+10 n+16, \\
N(3, n, 1)=n^{4}-7 n^{3}+16 n^{2}+24 n+20, \\
N(4, n, 1)=n^{5}-9 n^{4}+24 n^{3}+32 n^{2}+28 n+24, \\
N(5, n, 1)=n^{6}-11 n^{5}+34 n^{4}+40 n^{3}+36 n^{2}+32 n+28, \\
N(6, n, 1)=n^{7}-13 n^{6}+46 n^{5}+48 n^{4}+44 n^{3}+40 n^{2}+36 n+32, \\
N(7, n, 1)=n^{8}-15 n^{7}+60 n^{6}+56 n^{5}+52 n^{4}+48 n^{3}+44 n^{2}+40 n+36 .
\end{gathered}
$$

The regular structure of these polynomials prompts us to make the following conjecture: 
Conjecture 1. For each $n \geq 3$ and each $\mu \in[1, n-2]$, the value of $N(\mu, n, 1)$ is given by the polynomial $n^{\mu+1}-(2 \mu+1) n^{\mu}+\left(\mu^{2}+\mu+4\right) n^{\mu-1}+\sum_{i=0}^{\mu-2}(4 i+4 \mu+8) n^{i}$, where it is understood that this expression reduces to $n^{2}-3 n+6$ when $\mu=1$.

Similarly, analysing the values taken by $N(\mu, n, 2)$ for different values of $n$ and $\mu$ leads us to make the following conjecture:

Conjecture 2. For each $n \geq 4$ and each $\mu \in[2, n-2]$, the value of $N(\mu, n, 2)$ is given by the polynomial $2 n^{\mu}+2 n^{\mu-1}-\sum_{i=1}^{\mu-2}\left(4 i^{2}+2 \mu^{2}-4 \mu i+12 i+2 \mu+6\right) n^{i}-\left(2 \mu^{2}+8 \mu+8\right)$, where it is understood that this expression reduces to $2 n^{2}+2 n-32$ when $\mu=2$.

Finally, motivated by the results of our computer-aided enumerations, we make a more general conjecture concerning the behaviour of $N(\mu, n, \Delta)$.

Conjecture 3. For each $n \geq 4$, each $\mu \in[1, n-2]$, and each $\Delta \in[1, \mu+1]$, the value of $N(\mu, n, \Delta)$ follows a polynomial in $n$ (when $\mu$ and $\Delta$ are held fixed and $n$ varies). Conjectures 1 and 2 specify the polynomials for $N(\mu, n, 1)$ and $N(\mu, n, 2)$ respectively. For each $\Delta \in[3, \mu+1]$, the value of $N(\mu, n, \Delta)$ follows a degree- $\mu$ polynomial in $n$, with the two leading terms of the polynomial having the following form: $2 n^{\mu}-2 n^{\mu-1}$ for $\Delta=3$; $2 n^{\mu}+(2 \mu-4 \Delta+10) n^{\mu-1}$ for each $\Delta \in[4, \mu]$; and $2 n^{\mu}-\left(\mu^{2}+3 \mu-2\right) n^{\mu-1}$ for $\Delta=\mu+1$.

Conjecture 3 is consistent with the fact that $\sum_{\Delta=1}^{\mu+1} N(\mu, n, \Delta)=n^{\mu}(n-1)$, as the coefficients of $n^{\mu+1}, n^{\mu}$, and $n^{\mu-1}$ in this sum are $1,-1$, and 0 if Conjecture 3 is true.

The authors strongly suspect that all three conjectures presented above will turn out to be true, but no rigorous proofs have yet been found.

\section{Two consequences of Conjecture 1}

In this section, we show that if Conjecture 1 is true, then two interesting conjectures made by Thompson [24] are also true.

\subsection{The 'one-third conjecture'}

Let $n \geq 3$ be fixed. Suppose that a Prüfer string $P$ is selected uniformly at random from $\mathcal{P}_{n}$, and experiences a random mutation such that the mutation position $\mu$ is selected uniformly at random from the set $[1, n-2]$, and the value of $p_{\mu}^{\star}$ is selected uniformly at random from the set $[1, n] \backslash\left\{p_{\mu}\right\}$. Then, the probability $\rho(n)$ that this mutation is perfect (i.e., causes exactly one edge change in the corresponding tree) is readily seen to be

$$
\rho(n)=\frac{1}{n-2} \sum_{\mu=1}^{n-2} \frac{N(\mu, n, 1)}{n^{\mu}(n-1)} .
$$

Thompson ([24], p. 195) conjectured that, under the Prüfer Code, the asymptotic probability that a random mutation event is perfect is one-third; that is, $\lim _{n \rightarrow \infty} \rho(n)=\frac{1}{3}$. We now establish that Thompson's 'one-third conjecture' is true if Conjecture 1 is true. 
Theorem 4. If Conjecture 1 is true, then $\lim _{n \rightarrow \infty} \rho(n)=\frac{1}{3}$.

Proof. Under the assumption that Conjecture 1 is true, we can rewrite the formula for $\rho(n)$ as follows:

$$
\rho(n)=\frac{1}{(n-2)(n-1)} \sum_{\mu=1}^{n-2}\left(n-(2 \mu+1)+\left(\mu^{2}+\mu+4\right) n^{-1}+n^{-\mu} \sum_{i=0}^{\mu-2}(4 i+4 \mu+8) n^{i}\right) .
$$

Then, using elementary summation identities, it is easy to show that

$$
\sum_{\mu=1}^{n-2}(n-(2 \mu+1))=0
$$

and that

$$
\sum_{\mu=1}^{n-2}\left(\left(\mu^{2}+\mu+4\right) n^{-1}\right)=\frac{(n-1)(n-2)(2 n-3)}{6 n}+\frac{(n-1)(n-2)}{2 n}+\frac{4(n-2)}{n} .
$$

Note also that, for each $\mu \geq 2$,

$$
\sum_{i=0}^{\mu-2}\left((4 i+4 \mu+8) n^{i}\right)=4\left(\frac{(\mu-2) n^{\mu}-(\mu-1) n^{\mu-1}+n}{(n-1)^{2}}\right)+(4 \mu+8)\left(\frac{n^{\mu-1}-1}{n-1}\right),
$$

and it therefore follows that

$$
\begin{aligned}
\sum_{\mu=2}^{n-2} n^{-\mu} \sum_{i=0}^{\mu-2}\left((4 i+4 \mu+8) n^{i}\right)= & \frac{4}{(n-1)^{2}} \sum_{\mu=2}^{n-2}\left((\mu-2)-(\mu-1) n^{-1}+n^{1-\mu}\right) \\
& +\frac{4}{n(n-1)} \sum_{\mu=2}^{n-2}(\mu+2)-\frac{4}{n-1} \sum_{\mu=2}^{n-2}\left((\mu+2) n^{-\mu}\right) \\
= & \frac{4}{(n-1)^{2}}\left(\frac{(n-3)(n-4)}{2}-\frac{(n-2)(n-3)}{2 n}+\frac{1-n^{3-n}}{n-1}\right) \\
& +\frac{4}{n(n-1)}\left(\frac{(n-3)(n+4)}{2}\right)-\frac{4}{n-1}\left(\frac{4 n-3-n^{5-n}}{n(n-1)^{2}}\right) .
\end{aligned}
$$

Putting these results together, we see that

$$
\begin{aligned}
\rho(n)= & \frac{1}{(n-2)(n-1)}\left[\frac{(n-1)(n-2)(2 n-3)}{6 n}+\frac{(n-1)(n-2)}{2 n}+\frac{4(n-2)}{n}\right. \\
& +\frac{4}{(n-1)^{2}}\left(\frac{(n-3)(n-4)}{2}-\frac{(n-2)(n-3)}{2 n}+\frac{1-n^{3-n}}{n-1}\right) \\
& \left.+\frac{4}{n(n-1)}\left(\frac{(n-3)(n+4)}{2}\right)-\frac{4}{n-1}\left(\frac{4 n-3-n^{5-n}}{n(n-1)^{2}}\right)\right] .
\end{aligned}
$$

As $n$ tends to infinity, the first term of the righthand side tends to one-third, and the other terms tend to zero. Therefore, if Conjecture 1 is true, then $\lim _{n \rightarrow \infty} \rho(n)=\frac{1}{3}$. 


\subsection{The 'asymptotic curve conjecture'}

For any given $n \geq 3$, the valid range of $\mu$ is $[1, n-2]$. Suppose we map this range into the fixed real interval $[0,1]$ by setting $\alpha=\alpha(\mu, n)=\mu / n$. Further, suppose we define $\beta=\beta(\mu, n)=N(\mu, n, 1) /\left(n^{\mu}(n-1)\right)=\mathbb{P}(\Delta=1 \mid \mu)$.

Thompson ([24], p. 196) conjectured that the plot of $\beta$ against $\alpha$ (i.e., the piecewise linear graph that links together the $n-2$ points $\{(\alpha, \beta): \mu \in[1, n-2]\}$ in order) asymptotically approaches the curve $\beta=(1-\alpha)^{2}$ as $n$ tends to infinity. We now show that this conjecture is true if Conjecture 1 is true.

Theorem 5. If Conjecture 1 is true, then the plot of $\beta$ against $\alpha$, as described above, asymptotically approaches the curve $\beta=(1-\alpha)^{2}$ as $n$ tends to infinity.

Proof. If Conjecture 1 is true, then $\beta$ asymptotically approaches $1-\frac{2 \mu}{n}+\frac{\mu^{2}}{n^{2}}$ for large $n$. Since $\alpha=\mu / n$, this expression reduces to $1-2 \alpha+\alpha^{2}=(1-\alpha)^{2}$.

In closing, it is worth commenting on the relationship between Theorems 4 and 5 . Theorem 5 indicates that if the value of $\mu / n=\alpha$ is fixed, then the probability of perfect mutation tends to the value $(1-\alpha)^{2}$ as $n$ tends to infinity. However, if the value of $\mu$ is fixed instead, then the probability of perfect mutation tends to 1 as $n$ tends to infinity. On the other hand, Theorem 4 indicates that if the value of $\mu$ is distributed uniformly at random on the range $[1, n-2]$, then the probability of perfect mutation tends to the value one-third as $n$ tends to infinity; observe that this limiting value represents the area under the curve $\beta=(1-\alpha)^{2}$ between $\alpha=0$ and $\alpha=1$.

\section{Conclusion}

In this paper, we examined Algorithm D, a little-known decoding algorithm for the Prüfer Code, and showed that it possesses a number of remarkable properties. Not only is Algorithm D the simplest and most efficient method yet devised for decoding a Prüfer string into its corresponding tree, but it is also an online algorithm that takes advantage of the 'nested' nature of Prüfer strings. We then exploited Algorithm D to develop a number of novel results concerning the locality properties of the Prüfer Code, including three new conjectures.

In terms of future work, it would be valuable to try and establish whether our three conjectures are indeed true, and whether the enumeration results presented earlier in the paper contain any additional patterns of interest. The framework developed in this paper could also be used to investigate the locality of other tree representations in greater depth.

Finally, based on the findings in this paper, we recommend that researchers working with the Prüfer Code should deploy Algorithm D instead of existing decoding algorithms, as it is significantly more efficient and can bring about huge analytical simplifications. 


\section{References}

[1] S. Caminiti, I. Finocchi and R. Petreschi, "A unified approach to coding labeled trees." In Lecture Notes in Computer Science 2976: Proceedings of LATIN 2004 Theoretical Informatics (Buenos Aires, Argentina, April 2004), pp.339-348.

[2] S. Caminiti and R. Petreschi, "String coding of trees with locality and heritability." In Lecture Notes in Computer Science 3595: Proceedings of COCOON 2005 (Kunming, August 2005), pp. 251-262.

[3] A. Cayley, "A theorem on trees," Quarterly Journal of Mathematics, vol.23, pp. 376-378, 1889.

[4] H.-C. Chen and Y.-L. Wang, "An efficient algorithm for generating Prüfer codes from labelled trees," Theory of Computing Systems, vol. 33, pp.97-105, 2000.

[5] M. Cho, D. Kim, S. Seo, and H. Shin, "Colored Prüfer codes for $k$-edge colored trees," Electronic Journal of Combinatorics \#N10, 2004.

[6] N. Deo and P. Micikevičius, "Prüfer-like codes for labeled trees," Congressus Numerantium, vol. 151, pp.65-73, 2001.

[7] L. Devroye, Non-Uniform Random Variate Generation. New York: Springer-Verlag, 1986. Full text available at http://jeff.cs.mcgill.ca/ luc/rnbookindex.html

[8] T. Fleiner, "On Prüfer codes," ERGRES Technical Report No. 2005-16, 2005. Available online: www.cs.elte.hu/egres/tr/egres-05-16.pdf

[9] M. Gen and R. Cheng, Genetic Algorithms and Engineering Optimization. New York: Wiley, 2000.

[10] B. A. Julstrom, "Quick decoding and encoding of Prüfer strings: Exercises in data structures." Available online: http://citeseer.ist.psu.edu/326681.html

[11] B. A. Julstrom, "The Blob Code: A better string coding of spanning trees for evolutionary search." In Genetic and Evolutionary Computation Conference Workshop Program, pp. 256-261, San Mateo, California, 2001.

[12] P. Micikevičius, S. Caminiti, and N. Deo, "Linear-time algorithms for encoding trees as sequences of node labels." To appear in Congressus Numerantium, 2007.

[13] A. Nijenhuis and H. S. Wilf, Combinatorial algorithms for computers and calculators (Second edition). New York: Academic Press, 1978.

[14] C. C. Palmer and A. Kershenbaum, "Representing trees in genetic algorithms," 1994. Available online: http://citeseer.ist.psu.edu/palmer94representing.html

[15] T. Paulden and D. K. Smith, "From the Dandelion Code to the Rainbow Code: A class of bijective spanning tree representations with linear complexity and bounded locality," IEEE Transactions on Evolutionary Computation, vol. 10, no. 2, pp. 108-123, April 2006.

[16] T. Paulden and D. K. Smith. "Recent advances in the study of the Dandelion Code, Happy Code, and Blob Code spanning tree representations," In Proceedings 
of the 2006 IEEE World Congress on Computational Intelligence [DVD-ROM only], pp. 7464-7471, Vancouver, July 2006.

[17] T. Paulden and D. K. Smith, "Some novel locality results for the Blob Code spanning tree representation." In Proceedings of the 9th Genetic and Evolutionary Computation Conference (GECCO 2007), pp. 1320-1327, London, July 2007.

[18] S. Picciotto, "How to encode a tree," Ph.D. dissertation, University of California, San Diego, 1999.

[19] H. Prüfer, "Neuer Beweis eines Satzes über Permutationen," Archiv der Mathematik und Physik, vol. 27, pp. 742-744, 1918.

[20] P. Rao and B. Moon, "PRIX: Indexing and Querying XML Using Prüfer Sequences," Proceedings of the 20th International Conference on Data Engineering (ICDE'04), pp. 288-300, 2004.

[21] F. Rothlauf, Representations for Genetic and Evolutionary Algorithms (Second edition). Heidelberg, Germany: Physica-Verlag, 2006.

[22] F. Rothlauf and D. E. Goldberg, "Pruefernumbers and genetic algorithms: a lesson how the low locality of an encoding can harm the performance of GAs." In Lecture Notes in Computer Science 1917: Proceedings of PPSN VI (Paris, France, September 2000), pp. 395-404.

[23] S. Seo and H. Shin, "A generalized enumeration of labeled trees and reverse Prüfer algorithm," 2005. Available online: arxiv.org/pdf/math/0601009. Due to appear in Journal of Combinatorial Theory (Series A), vol.114, no. 7, pp. 1357-1361, 2007.

[24] E. B. Thompson, "The application of evolutionary algorithms to spanning tree problems," Ph.D. dissertation, University of Exeter, U.K., 2003.

[25] E. B. Thompson, T. Paulden, and D. K. Smith, "The Dandelion Code: A new coding of spanning trees for genetic algorithms," IEEE Transactions on Evolutionary Computation, vol. 11, no. 1, pp.91-100, February 2007.

[26] D. B. West, Introduction to Graph Theory (Second edition). Upper Saddle River, NJ: Prentice-Hall, 2001.

[27] B. Y. Wu and K.-M. Chao, Spanning Trees and Optimization Problems. London: Chapman \& Hall, 2004. 